CIC. Cuadernos de Información y Comunicación ISSN: 1135-7991

http://dx.doi.org/10.5209/ciyc.64636

\title{
Investigación de audiencias: lost in transition
}

\author{
Manuel Javier Callejo Gallego ${ }^{1}$
}

Recibido: 6 de diciembre de 2018 / Aceptado: 27 de marzo de 2019

Resumen. El proceso de digitalización ha supuesto una auténtica revolución en el sistema de la comunicación mediada institucionalmente. La gran mayoría de medios de comunicación tienen dificultades para encontrar un modelo económico para subsistir. Puede decirse que, a falta de nuevos modelos, están anclados en prácticas de difusión masiva y centralizada que cabe considerar como básicamente cosa del pasado. Pues bien, lo mismo ocurre con algo tan básico a tal modelo centralizado como la investigación de audiencia. Tras describir cuál ha sido el papel de la investigación de audiencias en ese modelo de difusión masiva y cómo se ha mantenido, a pesar de las críticas emergidas desde los principales actores de ese modelo a su forma de funcionamiento, se establece una sintética panorámica de los retos que ha de afrontar la investigación de audiencias para adaptarse a la transformación digital, ya que el sistema de la comunicación mediada institucionalmente y su relación con el sistema de mercado han cambiado radicalmente.

Palabras clave: Digitalización medios, sistema de comunicación mediada, investigación de audiencias, modelo centralizado.

\section{[en] Audiences Research: Lost in Transition}

\begin{abstract}
The digitization process has brought about an authentic revolution in the media system. The majority of the known "old media" has difficulties in finding an economic model to survive. They are anchored in a massive and centralized broadcasting model that can be considered basically dead. The same goes for something as basic to such a centralized model as audience research. After describing what has been the role of audience research in this model of mass broadcasting and how it has been maintained, despite the criticisms that emerged to its way of working from the main actors of that model, a synthetic summary of the challenges that audiences research must to take in order to adapt to digital transformation.
\end{abstract}

Key words: Media digitalization, Mediated communication system, Audience research, Centralized model.

Sumario. 1. Introducción. 2. Eso que llamamos demandas de la industria: puntos críticos. 3. De los viejos problemas, a los nuevos retos. 4. Más allá de los debates sobre la medida, la recomposición del sistema de comunicación mediada. 5. A modo de conclusión: retomar el rumbo. 6. Referencias bibliográficas.

Cómo citar: Callejo Gallego, M.J. (2019). Investigación de audiencias: lost in transition, en CIC. Cuadernos de Información y Comunicación 24, 155-173.

1 Universidad Nacional de Educación a Distancia 


\section{Introducción}

La observación empírica en comunicación ha tendido a dar preferencia a la investigación de los mensajes. Con mayor o menor capacidad de registro, según los medios de comunicación y las sociedades, se encuentran a mano, archivados y de una manera relativamente disponible para su fácil acopio, o, al menos, sin infranqueables obstáculos para ser observados. Por otro lado, esta centralidad textual encuentra en los objetivos de certificar la representación de una realidad o de un discurso o ideología argumentos bastante directos para impulsar el esfuerzo empírico. En muchos de estos estudios con los mensajes en el centro se lleva a cabo una doble asunción. En primer lugar, se asume que el mensaje es asumido como lo interpreta el observador, que es la crítica que ácidamente formuló Umberto Eco (1982) hace ya casi cuarenta años. Incluso los Estudios Culturales, que pasan por ser el revulsivo renovador en el estudio de la comunicación desde los receptores, estaban más enfocados a explicar el texto (Nightingale, 1996:43), que a dar cuenta de los procesos de acumulación de las audiencias sobre tales textos, pudiéndose derivar de su investigación empírica que era el propio texto la exclusiva razón de la acumulación de audiencias a su alrededor. Ahora bien, si se tienen en cuenta los últimos cincuenta años, no parece exagerado decir que el estudio de las audiencias tiene un lugar central en la investigación de la comunicación, tanto básica como, sobre todo, en la investigación aplicada. Basta recordar la relevancia que adquirieron los estudios sobre los efectos de los orígenes de la televisión en la infancia o el hecho de que corrientes enteras de investigación-Usos y Gratificaciones o los propios Estudios Culturales- cabe identificarlas con el estudio de las audiencias y la recepción. Pero, sobre todo, lo reseñable es cómo este tipo de investigación enraizó en el centro de la industria del entretenimiento en general y de los medios de comunicación en particular. Es más, precisamente por la presión de la demanda derivada de la industria comunicacional sobre este campo de investigación (Autor, 1995; Bermejo, 2007) fue la que antes tomó conciencia de la necesidad de renovarse tecnológica y metodológicamente ${ }^{2}$ con la irrupción de internet. Otra cuestión es si, a pesar de esta toma de conciencia, ha sido capaz de renovarse al exigente ritmo de la demanda de la propia industria. Pues bien, el presente trabajo aborda los principales retos con los que actualmente se enfrenta la investigación de audiencias aplicada, siendo retos tanto de carácter teórico o conceptual, como de orden metodológico.

\section{Eso que llamamos demandas de la industria: puntos críticos}

No puede decirse que la investigación de audiencias goce actualmente de buena salud. En distintos foros, especialmente profesionales, se ha extendido la sensación de que la industria genera unas demandas que, al menos, la tradicional forma de dar cuenta de la audiencia es incapaz de responder. Como si hubiera perdido el tren en la transición a una industria tan digitalizada, que hasta el mismo atributo queda ya un tanto anticuado. Si en un interesante volumen, la profesora Amparo Huertas (2015) da oportuna cuenta del estado en que se encuentran los desarrollos de los estudios de

2 Siguiendo la estela de Bachelard, Bourdieu e Ibáñez, se plantean cuatro niveles de la investigación: ontológico, epistemológico, metodológico y tecnológico. Véase Autor (2009). 
la audiencia y la recepción, así como la necesidad de dar un giro hacia el compromiso social, aquí se reflexiona sobre las condiciones estructurales en las que se encuentra este tipo de investigación, sin perder la vista sobre la necesidad señalada.

Hablar de la necesidad de dar respuestas a las demandas de la industria -y, en definitiva, del mercado, entendido en un sentido amplio- conlleva que el sistema social del mercado y el sistema social de la ciencia pueden articular acciones conjuntas. Hay demandas de la industria que incentivan la acción de las instituciones de conocimiento, como hay propuestas desde las instituciones de conocimiento que derivan en beneficios industriales (Burke, 2017). Pero, a la vez y al menos en este campo, la continua experiencia de dos mundos distintos. Cuando desde la academia se abordan los receptores se habla preferentemente de conceptos como identidad, representaciones, inclusión/exclusión social, estilos de vida, etc. Cuando se hace desde la industria, se habla principalmente de targets, expectativas, visitas, audiencias cautivas, etc. Hay veces que esos mundos que se relacionan con los medios de comunicación y sus mensajes parecen distintos, según lo vea un académico o un ejecutivo de las industrias culturales. En el ámbito de las ciencias sociales, investigación académica e investigación para el mercado parecen haber emprendido caminos divergentes. Mientras que la primera se encuentra en plena reflexión y reflexividad, especialmente bajo los paradigmas del constructivismo y de la acción-participativa, lo que podríamos denominar el giro participativo ${ }^{3}$; la investigación de mercado se mantiene enraizada en el positivismo y, tal vez por ello en la investigación de audiencias, se ha ido acercando a las disciplinas en las que domina un enfoque netamente positivista, como las neurociencias, y así los estudios sobre reacciones impulsivas de la audiencia, fundamentadas en bases neurológicas (Vecchiato et al., 2013) y, así, recuperando viejos fantasmas de manipulación inconsciente o subconsciente (Packard, 1957); o, de manera solo aparentemente opuesta, aun cuando también recuperando viejas propuestas de modelos racionales de la atención ( $\mathrm{Si}$ mon, 1996), o, por otro lado, las ciencias matemáticas y de la inteligencia artificial (machine learning: Domingos, 2015) como base disciplinar y teniendo los big-data como principal materia prima, que, como advierten críticamente autores como Cathy O'Neil (2016), pueden convertirse en fuentes de desigualdad y amenazas para la democracia. Así, si utilizamos el socorrido término de lo social, podría decirse que la investigación académica es la que en la actualidad corre con él; mientras que la investigación para la industria parece haberlo arrinconado en favor del recurso al algoritmo o el registro de las supuestas activaciones neuronales.

Ha de reconocerse que la medición de audiencias ha tendido a ser mirada con sospecha desde la sociología académica casi desde sus principios ${ }^{4}$; y ello a pesar de las notables contribuciones metodológicas que ilustres investigadores como Lazarsfeld ${ }^{5}$ y sus colaboradores llevaron a cabo, como la del propio panel (Lazarsfeld et al., 1944). La investigación académica se interesó por: la influencia, los efectos, la

3 Véase, a modo de punta de lanza próxima al marco de este artículo, el panorama descrito por Noortje Marres (2017).

$4 \quad$ Valga el ejemplo de Adorno y Horkheimer (1971), como referente crítico en frontal oposición a la investigación que denomina administrativa frente a la investigación crítica.

5 Son numerosas las contribuciones de este autor, destacamos aquí sólo algunas: Lazarsfeld (1940, 1941, 1950, 1972), Lazarsfeld y Kendall (1948), Lazarsfeld y Merton (1948) o las series que coordinó junto a Stanton (1942, 1944 y 1949). Como pertinente y justamente plantea Katz (1987), la disciplina de la investigación en comunicación fue creada por Paul F. Lazarsfeld. 
"agenda" como marco de representaciones de lo político o lo importante, la cultivation, la hegemonía, la dominación, la generación de estereotipos, la exclusión social, las culturas de los receptores, el poder de la audiencia, etc... ¿Y la medida? Como un asunto secundario, relativamente instrumental ${ }^{6}$. De medir algo, ha habido más interés en medir lo que los medios hacían a la audiencia o, más concretamente, lo que no conseguían hacer, retomando el clásico trabajo de Klapper (1974), que lo que la audiencia hace a los medios o a la propia audiencia, como de forma tan certera plantea Umberto Eco.

Desde la perspectiva del nivel tecnológico, que es el que adquiere preferencia entre la demanda desde la industria de los medios de comunicación, las dos principales preguntas que la han estructurado la investigación empírica son:

- ¿Cómo se recoge eso que llamamos audiencia? ¿Cómo se producen los registros?

- ¿Cómo se mide?

Aunque próximas en sentido, no se trata de la misma pregunta, aun cuando ambas cuestiones hacen especial hincapié en lo procedimental, frente a los objetivos. Y es que el objetivo compartido es la medición, en cuanto la obtención de un número de seguidores de los mensajes -o del medio de comunicación, en su conjunto- para ser comparado con el número obtenido por otros mensajes u otros medios de comunicación. Puesto que la medida está destinada a la comparación, se requieren procedimientos compartidos, tanto por los que son objeto de la medición, como, sobre todo, por quienes son receptores últimos de tal medición, para, a su vez, tomar sus medidas, es decir, decisiones sobre inversiones y emplazamientos de publicidad.

El cómo se responde a una u otra pregunta parece central para entender la lógica del sistema de comunicación mediada institucionalmente en cada momento (Napoli, 2011:287) y, en definitiva, qué se entiende por audiencia y qué comportamientos interesan de la misma. Al menos, lo que interesa desde el punto de vista de la generación de valor económico. Pues bien, hasta la definitiva extensión de la web 2.0 y la expansión de la comunicación que esta ha supuesto, incluyendo la propia actividad comunicativa de los, hasta entonces, receptores, la cuestión venía respondida por el oportuno diagnóstico de Dallas Smythe (1983): la audiencia como mercancía que los medios de comunicación venden a los anunciantes. De aquí la fuerte asimilación entre investigación de audiencia y medición de la audiencia. La medición es tal prioridad que se llega a afirmar el lugar central que ocupa la medición de audiencias en el sistema de comunicación institucionalizada: "la preocupación por medir la audiencia, por cuantificar el mercado, nace junto con los medios" (Huertas, 2002:56)

Los más o menos consensuados estándares de medición ${ }^{7}$ están destinados a certificar (Autor, 2000) el monto de audiencia obtenido por los productos: mensajes, especialmente tomando la forma de programas para televisión o radio; o cabeceras, para los medios de comunicación periódicos escritos. Para los anunciantes y para los

6 Reivindicado por una perspectiva psicológica experimental que intenta abrirse espacio a partir de una retórica que recuerda bastante al empirismo abstracto denunciado por Wright Mills (1999).

7 Estándares de medición para la certificación semejantes a los que, en ese mismo período, se establecían en otros campos empresariales, como pone de manifiesto el surgimiento de entidades certificadoras, ya sea para certificar la validez de los productos (normas ISO), ya para puntuar y clasificar a las empresas que ofrecían productos y, crecientemente, servicios (EFQM, por ejemplo). 
medios de comunicación ${ }^{8}$, el centro está en la constatación de audiencia que, a su vez, genera esos indicadores, que toman la denominación de ratings, es decir, de indicadores que sirven para calibrar el lugar de cada mensaje-programa-medio de comunicación a lo largo del día. Por lo tanto, a lo largo de la programación. De hecho, puede decirse que si la programación podía entenderse como el esquema del flujo televisivo (Williams, 1974), una de las obsesiones de la investigación de audiencia estaba en la observación de los flujos de la audiencia, entendidos como los movimientos por parte del total de los elementos de la audiencia entre las distintas ofertas en cada uno de los tipos de medios de comunicación, siendo la televisión el más enfocado desde esta perspectiva ${ }^{9}$. Representado el conjunto de programaciones ofrecidos por las distintas concreciones o instituciones de un mismo tipo de medio de comunicación -su ilustración más pertinente corresponde a la televisión- como un entramado de tuberías, la audiencia se constituye en el líquido que ha de canalizarse hacia la propia oferta.

Tales objetivos para la investigación de audiencias parten principalmente de una doble demanda de los anunciantes. Por un lado, la demanda de servicio y actuación a los medios de comunicación para hacer llegar sus mensajes -información, publicidad- a los potenciales consumidores. Por otro lado, de obtención de ciertos retornos sobre el cumplimiento de tal servicio, con cada vez mayores exigencias sobre los detalles del servicio contratado: detalle y segmentación de los perfiles de los receptores de los mensajes, precisión sobre si tal recepción de los mensajes se establecía, frente a la práctica de la evitación de la publicidad por parte de los receptores, especialmente tras la extensión del uso del mando a distancia, adopción técnica que fue muy temprana en países, como Estados Unidos, donde la ampliación de la oferta de

8 Se deja a un lado el importante papel que adquieren las empresas mediadoras entre anunciantes y medios de comunicación, como son las agencias de publicidad y el diseño de sus estratégicos planes de publicidad, siendo especialmente relevante el papel que adquieren las denominadas centrales de medios, determinando lo que se invierte por cada anunciante en cada medio, $\mathrm{y}$, sobre todo por su carácter oligopólico, consistiendo su principal negocio en comprar al por mayor grandes cantidades de espacio y tiempo publicitario de los medios de comunicación, a precios muy bajos, para revenderlos a los anunciantes. De hecho, está por hacerse el análisis del papel de estas entidades en la política económica del sistema de medios de comunicación, debiéndose rescatar la original aportación de Schiller (1983) al respecto, así como en el propio sistema político, ya que, por ejemplo, se constituyen en fuentes de ruido en la directa relación entre política y las decisiones de publicidad institucional, por un lado, y medios de comunicación, por el otro lado. Hay que tener en cuenta que la racionalidad estratégica de las centrales de medios o centrales de compra de espacios-tiempos publicitarios, vertebrada sobre estrategias de alcanzar de la forma más eficiente posible a los pertinentes perfiles de consumidores o votantes, no siempre coincide con la racionalidad estratégica de la política, especialmente cuando se encuentra interesada en que determinados medios de comunicación ofrezcan determinada construcción de la realidad. Tal vez esto explique el hecho de que estas centrales de medios se hayan constituido en ocasiones en preferentes destinos de las denominadas "puertas giratorias" de cargos políticos relacionados con la comunicación, cuando deciden "retirarse" de la política.

9 Hasta su cierre, la empresa de investigación de audiencias y asesoramiento en comunicación Corporación Multimedia tenía en el denominado videorating su producto estrella, teniendo como función ofrecer en cada momento lo que estaba viendo - o no viendo- el conjunto de la audiencia. Es decir, lo que se ofrecía era la distribución del total de la audiencia entre el conjunto de programaciones en un momento dado. La perspectiva distributiva en términos de Ibáñez (1979) era evidente. Ahora bien, la asunción de esta perspectiva parece asimilar su objeto - la audiencia- más a una especie de capital, que a esa mercancía se produce y vende con cada mensaje ofrecido por el medio de comunicación. Se asemeja más a un capital en stock, en bolsa, que los medios de comunicación intentan capturar para, así, cazar inversores-anunciantes. Señalar aquí solamente como este aparentemente sutil cambio en la representación de la audiencia aparece con la significativa multiplicación de oferta de canales televisivos que tiene lugar en Europa occidental en los primeros años del siglo XXI. Sobre la concepción de la audiencia como capital, véase Autor (2018). 
canales televisivos arraigó relativamente pronto, compitiendo por el mismo líquido (audiencia), contra la presión del mismo instrumento (mando a distancia); y, por ello mismo, desde contenidos prácticamente iguales y hegemónicos, como muestra Gitlin (2000) a partir de un trabajo con unas doscientas entrevistas a distintos actores de la industria norteamericana de los años setenta, de manera que el flujo de imágenes termina imponiéndose en el flujo de la audiencia (Gitlin, 2007).

Demandas de los anunciantes que, en buena parte, partían de las sospechas que tenían sobre la actuación publicitaria, no exenta de lo que podríamos denominar cierta mística carismática. Hay que tener en cuenta que buena parte de estos anunciantes proceden de una cultura eminentemente productiva, industrial ${ }^{10}$. Como cínicamente refleja uno de los grandes gurús de la publicidad de los momentos expansivos de la sociedad de consumo y de la expansión de los denominados medios de comunicación de masas, David Ogilvy, atribuyendo la frase a Lord Levelhulme: la mitad del dinero que gasto en publicidad es dinero perdido y el caso es que no sé qué mitad es (Ogilvy, 1984:80). Una sospecha constante que hace que la mayor parte de las respuestas que se han venido dando por parte de la industria de la investigación de audiencias estén trufadas de lo que puede tomarse como puntos críticos.

Mientras, los ejecutivos de los medios de comunicación hacen lo que pueden para obtener la inversión de los escépticos anunciantes, partiendo también de ellos las exigencias de medición y sobre la medición de audiencias. Hay que tener en cuenta las particularidades que ha de asumir el sistema de comunicación mediada cuando se asimila a un mercado en el que la mercancía principal es la audiencia. En un modelo en el que los medios de comunicación venden audiencia a los anunciantes de manera masiva, sin que quede registro alguno en los propios medios de comunicación del directo contacto entre los elementos de la audiencia y los elementos de contenido ofrecidos por el medio de comunicación (programas, noticias, reportajes o publicidad), la medida realizada y certificada por la investigación de audiencias es prácticamente el único instrumento para generar el precio por el servicio ${ }^{11}$ de captar consumidores para los anunciantes. Es decir y hasta que no llega la posibilidad de registros y auditoría de visitas, descargas, etc. de los sitios digitalizados de los medios de comunicación, las "ventas" apenas suponen su unidad de medida para los medios de comunicación de mayor inversión publicitaria, como televisión y radio ${ }^{12}$. La medida de la audiencia es la fuente del precio, de aquí las extraordinarias y continuas exigencias y disputas sobre la misma, muy distintas a las acontecidas en otros campos de la investigación social. Por ejemplo, un productor de bienes-objetos (mercancía) recibirá sus ingresos a partir del número de bienes-objetos que venda a determinado precio. Puede decirse que no requiere medir sino solo contar cada bien-objeto vendido. Desde la asunción de que la audiencia es el bien-objeto (mercancía), no siéndolo los mensajes o contenidos emitidos, supuestamente el medio de comunicación recibe sus ingresos de los anunciantes a partir del número de elementos de la audiencia que vende. Ahora

10 Estos encuentros entre la cultura industrial de los anunciantes y la cultura postindustrial -según la concepción de Daniel Bell (1973)- se encuentran magistralmente representados en series como Mad Men.

11 Sobre la producción social de los precios, véase el magnífico trabajo de Boltanski y Esquerre (2017).

12 No ocurre lo mismo con los diarios, vendidos en quiosco, o los medios de comunicación que requieren el paso por taquilla, lo que incluye a la propia televisión en la que se paga-por-ver. Pero incluso en los periódicos o en el pago-por-ver no se tiene directamente el número de unidades de la audiencia, ya que un mismo ejemplar del diario puede ser leído por varias personas, al igual que un programa de la televisión por pago puede ser visto por varias personas ante la pantalla. 
bien, desde la aceptación de este modelo: a) el anunciante no va pagando, uno por uno, por cada miembro de la audiencia obtenido; b) el precio se pone no tanto -aun cuando esto ha tenido distintas concreciones- por las unidades de audiencia obtenidas sino por las unidades de audiencia previstas; c) las unidades de audiencia obtenidas derivan directamente de los procedimientos e instrumentos de medida aceptados y que dependen de modelos estimación estadística. Es decir y para hacerlo más fácil, en la comunicación difundida a través de instituciones como los medios de comunicación, el intercambio entre medio de comunicación y anunciante, en el que el primero ofrece unidades de la mercancía audiencia al segundo, a cambio de dinero, realmente no existe, pues no hay registro de esas unidades. Tales unidades son creadas por los instrumentos de medición, de aquí la relevancia que adquieren éstos.

Desde la pregunta sobre cómo se producen los registros de esas unidades de la audiencia, la respuesta más extendida en el campo profesional ha estado centrada en la oferta de muestras estadísticamente representativas. La crítica general de la industria se ha fijado principalmente en la continua falta de tamaño, como crítica general $^{13}$, lo que, dada la creciente heterogeneidad de las sociedades, conlleva la crítica a la infrarrepresentación de determinadas subpoblaciones, lo que tiende a debilitar su representatividad estadística hasta niveles de un error muestral que complica cualquier afirmación sobre las mismas, o de circunstancias de recepción. Así, una repetida crítica presente en los encuentros propiciados por la Asociación Española de Estudios de Mercado, Marketing y Opinión (AEDEMO) ha estado centrada en la incapacidad de importantes y relevantes estudios, como el EGM, para dar cuenta de las audiencias locales, algo de estratégica relevancia para los medios de comunicación que actúan en tal contexto territorial. El otro tipo de crítica ha estado más dirigido a los sistemas de medición y certificación de las audiencias televisivas. Así, se ha intentado ir superando, como si fuera una especie de carrera de obstáculos, situaciones que aparecían como perdidas, como constatación de una audiencia existente, pero que inicialmente se escapaba a la medición: segundas residencias, segundos televisores, posesión de aparatos complementarios (vídeo), seguimientos asíncronos...), los comentarios sobre los programas en los social media, etc.

El otro conjunto de puntos críticos en los procesos de registro ha estado relacionado con el reclutamiento de los participantes. Se trata de una crítica presente en todas las operaciones de observación mediante encuesta con cuestionario estandarizado con diseño muestral no aleatorio; pero que se acentúa en un tipo de diseño metodológico, el longitudinal mediante panel, que ha estado destinado a certificar las audiencias de industrias audiovisuales, como la televisiva o la radiofónica ${ }^{14}$. Las principales críticas consisten en:

- Cansancio habitual de los panelistas, lo que tiene como reacción la necesidad de acelerar la rotación de las unidades. Este aspecto afecta directamente a la calidad del panel, ya sea por la proyección en la forma de responder de los que se encuentran cansados, ya sea desde los desajustes que siempre se producen en la sustitución de las unidades, unos desajustes que pueden acumu-

13 Especialmente en operaciones bien conocidas, como los estudios mediante panel de audímetros o el Estudio General de Medios, como estudio multimedio que desarrolla en España la AIMC.

14 Una crítica que quedó reforzada a partir de la revelación por parte del diario ABC de los registros de las direcciones de los hogares panelistas de la empresa Ecotel en enero del 1991. 
larse, si las sustituciones se llevan a cabo en el tiempo acelerado que requiere mantener la máquina de la medición continua en funcionamiento. Hay que tener en cuenta que, si bien el cansancio de los informantes es uno de los problemas de todos los estudios que utilizan el diseño de panel, la recogida diaria de información, exigida por algunos estudios de audiencia, aceleran esta generación de cansancio.

- La mayor parte de los diseños panel desarrollados por el sector privado ${ }^{15}$ remuneran directa o indirectamente la participación de los encuestados. Tal característica es habitual en los paneles realizados para el estudio de la audiencia de los medios de comunicación. La remuneración amenaza la representatividad de la muestra, como por ejemplo y más recientemente ocurre en los paneles online utilizados para las encuestas, donde empieza a aparecer la figura del "informante o encuestado profesional", que contesta a todo, sumando pequeñas retribuciones recibidas como pago -realmente pueden considerarse micropagos por sus pequeños importes- por completar cada cuestionario, teniendo en cuenta que las gratificaciones por trabajar para un panel-regalos, gratuidad de la línea telefónica que recoge la información de los audímetrosse hacen atractivas para algunos perfiles sociales. Si está "mal remunerada" la contribución al panel, regresamos al problema de la rotación acelerada. En ambos casos y como se pone de manifiesto en distintos estudios, el hecho del pago implica la asunción de un tipo de trabajo, al que están más dispuestas unas categorías sociales que otras. ¿Son iguales los que hacen el esfuerzo de participar voluntariamente en un panel, de los que no?

- Las tecnologías de la recogida afecta al comportamiento de las audiencias observado, generando reactividad. De hecho, el debate entre audímetros activos, que requieren la consciente implicación de los participantes, y audímetros pasivos todavía está presente.

- La necesidad del secreto de las unidades del panel, para que los medios de comunicación evaluados no puedan ponerse en contacto con la muestra, hace que su control externo sea difícil, requiriendo ocasionalmente la mediación de figuras parecidas a las de los fedatarios públicos.

- Cuando la observación continua se lleva a cabo sin reclutamiento explícito de los participantes, sin muestra, con carácter censal, como ocurre en la observación desde los "ofertantes de servicio": server log analysis, plataformas, etc., las críticas se centran en:

- Las dificultades para afinar en los perfiles sociodemográficos, quedándose constreñido el análisis al seguimiento de la actividad: reducción de la medición al número de visitas o de aparatos receptores que han seleccionado un producto. ${ }^{16}$ En todo caso, hay que tener en cuenta que

15 Aun cuando fuera del estricto campo de los estudios de comunicación, hay que señalar que otra cosa son las operaciones llevadas a cabo por instituciones públicas, como el Centro de Investigaciones Sociológicas o el Instituto Nacional de Estadística, que no remuneran a los informantes, si bien existe una particular diferencia entre ambas instituciones y es que la participación en la mayor parte de las operaciones de la segunda la participación de los informantes seleccionados es obligatoria, según la Ley 12/1989, de 9 de mayo, de la Función Estadística Pública.

16 Sobre el cambio en la generación del sistema de variables a observar que se está produciendo al pasar del seguimiento del comportamiento de las unidades de la audiencia a la predicción algorítmica de los futuros comportamientos de la audiencia, como ocurre en Netflix, véase Finn (2017). 
la audiencia queda compuesta principalmente por aparatos ( $\mathrm{pc}$, teléfono móvil o televisores) a los que de manera real o simbólica se conecta el dispositivo de medición; pero realmente no por sujetos.

- La crítica ética a la transgresión de la privacidad que se produce, ya que la observación se hace sin el consentimiento explícito de las unidades observadas y, la mayor parte de las veces, sin conciencia de éstos de estar siendo observados ${ }^{17}$.

Teniendo en cuenta los objetivos, ofrecer medidas justas para los medios de comunicación y los anunciantes, no todos los problemas han venido teniendo el mismo peso. De hecho, aquellos que tenían que ver con posibles atentados a la privacidad de los informantes o de las unidades observadas, quedan en un muy distante lugar de la obtención de datos con la mayor apariencia de validez y fiabilidad. Lo que era justo para los demandantes de la investigación se superponía a lo que es justo para los observados.

\section{De los viejos problemas, a los nuevos retos}

Puede decirse que buena parte de esos problemas se mantienen a lo largo del tiempo, incluso a pesar de los esfuerzos de la industria de investigación de audiencias por superarlos y responder de una manera más adecuada a las demandas de anunciantes y medios de comunicación. Cabe hablar así de un compromiso de la industria de la investigación por mejorar el modelo y, sobre todo, para realizar los cambios suficientes para mantenerlo. Puede decirse que, en todo momento y lugar y a pesar del necesario consenso para el establecimiento de sistemas y estándares de medición-certificación de las audiencias, la sospecha sobre tales sistemas y estándares se ha sostenido, lo que explica en parte la constante renovación de los mismos: aumentos de tamaños muestrales, incorporación de instrumentos digitales para la recolección y registro de información de manera autocumplimentada, incorporación de los distintos aparatos receptores del hogar, reducción al segundo de los tiempos de registro, etc., haciendo de la investigación de audiencias uno de los campos líderes en la innovación metodológica en el conjunto de las ciencias sociales. Sin embargo, contrasta el enorme esfuerzo empírico y, en cierta forma, metodológico, con la escasa atención dada desde la industria de la investigación de audiencias a la reflexión teórica y, en especial, al debate sobre lo que se entiende como audiencia y, sobre todo, la continuación de la sospecha. Ambos aspectos se alimentan entre sí, ya que lo que parece subyacer es la sospecha sobre el modelo de los ratings de audiencia, en él ésta es una especie de ente invisible derivado de procedimientos que cuesta entender a los no expertos o legos, como los procedimientos derivados de la estimación estadística. En la industria de los medios, el paso desde una muestra que tiene a ser siempre considera pequeña en su tamaño y el total de la población, constituye parte esencial de un modelo que se ha concebido menor con respecto a la potencial alternativa de registrar y contabilizar directamente a los que entran en contacto con los mensajes, dejando huella de este acontecimiento (Carr, 2011; Finn, 2017), retomando el concepto comtiano de ingeniería social (Hadnagy, 2010; Mitnick, 2011). Si el modelo de la estimación se ha mantenido durante tantos años, a pesar del difícil equilibrio en el

17 A ello, habría que añadir el peligro de "virus" que afecten los logs. 
que se encontraba, ha sido por la convergencia de intereses estructurales de medios de comunicación, anunciantes, empresas de marketing y diseñadores de publicidad.

A los viejos objetivos y problemas, se añaden los nuevos derivados de lo que podemos denominar la intensificación de la digitalización de la sociedad en general y del sistema de medios de comunicación en particular. Así, a la necesidad de seguir a la audiencia a través de las distintas programaciones, surgen distintos objetivos y necesidades de la demanda como consecuencia de lo que puede describirse como multiplicación digital del sistema de medios de comunicación, lo que se concreta en:

- Multiplicación de la oferta, a partir de la multiplicación de canales, medios y dispositivos, lo que genera, a su vez, una multiplicación de preguntas de investigación: ¿son los mismos o distintos los que siguen contenidos en uno $\mathrm{u}$ otro dispositivo o medio de comunicación? ¿es un tipo de audiencia distinta en cada uno de ellos? ¿se ven los mismos anuncios? ¿se ven de la misma manera?

- Multiplicación de la capacidad interactiva de la audiencia, de la actuación de los receptores de los mensajes con respecto a los mimos, ya sea a partir de: a) interactividad en el propio medio que ha publicado el mensaje; b) interactividad extra-medio, con otros medios de comunicación, pero a partir del comportamiento como audiencia con respecto al mensaje de uno de esos medios, como ocurre en el tráfico de mensajes interactivos que se produce en las redes sociales alrededor de la emisión de, por ejemplo, un evento o programa televisado. La industria llama la atención sobre la conveniencia de registrar, ordenar e implicar estos mensajes en el análisis, en la medida que cualifican la audiencia y la recepción ${ }^{18}$. La acción de la audiencia sobre los mensajes de los medios va bastante más allá del esfuerzo de dedicación de tiempo que planteaba Smythe (1981), y, así, producción y reproducción de nuevas audiencias, como apunta Jenkins (2010; Jenkins, Ford y Green, 2015).

- Multiplicación de los canales o soportes a través de los que seguir un mismo contenido, programa o mensaje. En la práctica y especialmente para las categorías más jóvenes de la sociedad, esta multiplicación ha roto con la vinculación con la programación y, por lo tanto, con la necesidad de ajustar el seguimiento de un contenido a un espacio y un tiempo, de manera que ese mismo contenido puede seguirse en distintos lugares, ya no necesariamente ceñidos al hogar, y en cualquier momento ${ }^{19}$.

- Multiplicación de seguimiento simultáneo de distintos contenidos en un mismo medio de comunicación, a partir de aparatos que permitan, por ejemplo, varias pantallas. Ha de recordarse que una de las formas de observación de la audiencia mediante panel de audímetros se limitaba a registrar la sucesiva conexión con los distintos canales; pero no se recogía la posibilidad de simultánea conexión a dos o más canales.

18 De hecho, el análisis de los mensajes en los social media o en foros en internet, provisto por algunas empresas de la industria de la investigación de mercado, opinión pública y audiencias, se ha convertido en una especie de sucedáneo sustitutorio de la investigación cualitativa de audiencias destinada a recoger el sentido que el público daba a programas televisivos y la relación con los mismos.

19 La recuperación de las series televisivas se ha vinculado a la posibilidad de seguir varios episodios de un mismo título de una tacada, sumándose así el al carro del éxito de reputación de una serie, pudiéndose visionar los episodios iniciales, y sin tener que esperar a la emisión del episodio siguiente a una hora días determinados. 
A las anteriores multiplicaciones que pueden calificarse de carácter técnico, en cuanto derivadas del desarrollo de las posibilidades técnicas de emisión y recepción, cabría añadir otras paralelas, más directamente vinculadas a procesos sociales. Por un lado, la multiplicación de los criterios de categorización de la sociedad y, por ende, de la audiencia, donde las tradicionales variables sociodemográficas -como género, edad, nivel de estudios e incluso clase social- parecen quedarse cortas en favor de otras categorías que hacen mayor referencia a actitudes y comportamientos en el tiempo de ocio, como son las relacionadas con los estilos de vida, o directamente a otros comportamientos de consumo. En todo caso, una sociedad más diferenciada en todos los sentidos y niveles (Luhmann, 1990), en la que las anteriores categorías de diferenciación y segmentación aparecen con notables límites para llevar a cabo la explicación de complejos procesos y prácticas sociales en continua auto-referencia, como la de la relación con los medios de comunicación. Por otro lado, la multiplicación de instituciones medidoras, ofreciendo múltiples sistemas de medición o múltiples muestras-panel-censo, con lo que conlleva de tensión sobre el consenso para la aceptación de una única fuente para certificar lo que se certificaba antes, como era el número de audiencia acumulada por un medio de comunicación o mensaje en un momento determinado, algo que servía a los medios de comunicación tanto para predecir o, al menor, diseñar expectativas sobre el monto de audiencia que podía tener la emisión futura de un determinado mensaje o programa, bajo la aceptación del modesto modelo por el cual, si se había tenido tanta audiencia en la emisión de ese tipo de mensaje, era esperable una cantidad semejante para la siguiente emisión. Expectativas certificadas por los estándares de medición consensuados que, a su vez, servían a los medios de comunicación para poner precio a sus espacios de inserción publicitaria. Esta afirmación conlleva ya una pequeña corrección a la afirmación del referido Smythe (1983), pues lo que venden los medios de comunicación a los anunciantes no es la cantidad de audiencia sino la expectativa de la cantidad de audiencia ${ }^{20}$, lo que ha de tomarse como una considerable fractura a la consideración de la audiencia como mercancía. Pero la elaboración de las bases para tales expectativas utilizando los mismos procedimientos, en un contexto atravesado por los procesos de multiplicación apuntados, parece cada vez más difícil.

Un proceso general de multiplicación que afecta radicalmente a la manera de entender la audiencia: su forma, sus consistencias, incluso las evocaciones materiales como tal concepto. Parece ponerse en evidencia que, bajo el mismo término, se han venido refiriendo distintas calidades materiales a partir principalmente de cuáles eran los medios de comunicación de referencia y, sobre todo, de los procedimientos técnicos que se ponían en marcha para su registro y medición. Así, puede hablarse de:

- Audiencia sólida, como la resultante de la aplicación de los procedimientos de encuesta con cuestionario estandarizado a una muestra estadísticamente representativa de la población. La solidez deriva de distintas características vinculadas a estos procedimientos: a) los informantes son personas de carne y hueso; b) la representación de la audiencia como conjunto de personas con características sociodemográficas que acentúan la solidez de las mismas

20 Ya en una segunda fase, se extiende la contratación y ajuste de precios por audiencia certificada a posteriori, tras la emisión. 
(edad, sexo, nivel de estudios, hábitat, etc.); c) la mediación del lenguaje, de la verbalización (wording), en la recogida de la información sobre lo que el informante ha seguido en los medios de comunicación. Incluso la referencia a esta audiencia como masiva, va más allá de su acumulación en millones de unidades. Como masa, nos evoca cierta solidez, aunque manejable desde las intenciones de supuestos manipuladores, como ponía de relieve la aproximación desde la teoría de la aguja hipodérmica (Packard, 1957; García Matilla, 1990). La pregunta central a la que pretende responder la investigación de audiencia es sobre cuánta gente se pueden generar efectos, a cuántos se puede empujar al consumo.

- Audiencia líquida, resultante del interés, por parte de los medios de comunicación concretos, sobre cómo capturar unidades de audiencia a la competencia, presente en otros medios de comunicación. Consolidada ya la sociedad de consumo, parece asumirse que ya no se pueden generar más consumidores, incluidos los consumidores de medios, que alcanza a prácticamente la totalidad de la sociedad con la rápida extensión de la televisión en los hogares. Aparecen conceptos como trasvase, como paso del consumo de un ofertante a otro, o share o la parte de consumidores, entre todos los consumidores, que se asumían como propiedad de un ofertante. En consumo de bienes, de medios de comunicación o de votantes, lo importante es la observación de los flujos, de cómo se traspasaban "líquidos de un canal a otro"; pero siempre el mismo medio de comunicación o campo de consumo. El conjunto de la audiencia adquiere formas fluyentes y segmentadas, que han de ser embotelladas o embalsadas por los ofertantes, en una especie de circuito continuo. Se habla de "circuito de la comunicación de masas" (Deacon et al., 2011:313) en el que son las audiencias las que circulan entre programas y canales. A los procedimientos para la observación empírica y medida de estos flujos se les exige la perspectiva longitudinal, a ver a través de un tiempo capaz de registrar cambios, como ya apuntaba la inicial y paradigmática experiencia del panel desarrollada por Lazarsfeld et al. (1944). De una manera más rudimentaria y todavía con la mediación del cuestionario y, por lo tanto, del lenguaje, recogiendo las respuestas a las preguntas sobre los cambios, ya sea en la relación con los medios de comunicación, durante el día, como hacen los estudios de presupuestos temporales focalizados en un campo; ya sea preguntando directamente sobre las relaciones anteriores y presentes de los informantes con los medios de comunicación. Pero, sobre todo, los estudios de audiencia adquieren la forma panel, que permite hacer el seguimiento de los cambios de las unidades de la muestra, y cuya referencia principal en el campo de los medios de comunicación es el audímetro, que ya no requiere de la verbalización por parte del informante, pues parece bastar con la pulsación, adelantándose a la expansión del click, para dar cuenta de su relación con el medio de comunicación.

- La audiencia gaseosa se va posando en distintos sitios, en función de vientos difícilmente controlables y, de momento, difícilmente registrables y medibles, como ocurre con las vaporosas audiencias transmedias. Lo que circulan ahora son los productos en busca de audiencia de los más diversos tipos, en las más diversas posiciones y en disposición de diversos aparatos de recepción en distintos momentos y lugares. Precisamente porque es gaseosa, porque somos 
concebidos como cuerpos gaseosos, ya nadie escapa al hecho de ser audiencia. Antes, cuando la audiencia líquida, había tal vez la posibilidad de escapar de algunos medios, resistirse a ser audiencia a partir de la fuga que permitía un instrumento como el mando a distancia. Actualmente, cuando el aire que respiramos se ha convertido en mensaje mediado es imposible escapar a ser audiencia, algo de lo que tenemos evidencia en nuestras grandes ciudades con pantallas disponibles en cada rincón, en cada bolsillo. Terminales inteligentes que registran nuestros movimientos, la mayor parte de las veces sin que seamos conscientes de ello. Audiencia gaseosa a la que se activa y reactiva, que es soplada, con mensajes llegados a través de canales relativamente reconocidos -los miembros a los que hemos dado de alta en nuestros social mediadesde orígenes no siempre fácilmente identificables.

Como puede derivarse de lo anterior, tal vez por primera vez se esté ante una audiencia que aún no ha sido producida por instrumentos de medida. Una audiencia que aparentemente todavía no tiene instrumento que la mida de una manera integral. De momento y en la medida que los medios estén digitalizados se registran variables como: el número de conexiones a cada medio de comunicación, el tiempo de consumo/permanencia en cada una de las partes y el conjunto de ese medio de comunicación, los anuncios presentados durante ese tiempo a esa unidad de la audiencia, etc. En todo caso y frente a los anteriores procedimientos fundamentados en la estimación estadística y, por tanto, en el diseño de muestras para la observación, aquí nos encontramos con medidas a partir de registros derivados de toda la población que ha entrado en contacto con esos medios digitales. No se trata de muestras a partir de las que hay que establecer estimaciones sino del desarrollo de software a través del que hacer seguimiento de los movimientos y expresiones en los medios digitales en general y en los social media en particular (Kelleher et al. 2015; Barberá, 2015; van Zoonen et al., 2016).

El método del panel audimétrico parece haber llegado a un límite hace tiempo, parece haberse agotado. Sobre todo, se muestra incapaz de captar esa audiencia gaseosa, tal como ha venido estando diseñado, con relación a un único medio de comunicación; a la vez que, a través de la digitalización y por el solo hecho de estar recibiendo un flujo de mensajes, todos nos convertimos en miembros de un gran panel, en la medida que se registran nuestros cambios y movimientos. El panel que ahora podemos denominar tradicional -el de los audímetros situados en una muestra de hogares- ha estado bajo sospecha, como todo panel a partir de muestras que se extiende en el tiempo, pues se proyectaba la duda sobre la propia muestra: ¿quiénes eran esos sujetos que aguantaban día tras día las posibles molestias derivadas de tener un audímetro en casa y, en todo caso, el esfuerzo de formar parte de esa muestra?

El audímetro era el gran instrumento para medir y certificar las audiencias. A lo sumo, la discusión era sobre la concreción más conveniente del audímetro: activo o el pasivo, y se buscaron infinitas formas para que fuese pasivo. Se hablaba de la dictadura del audímetro, dictadura con respecto a los programas y la programación, dictadura en la medición. Hoy estamos ante un dictador zombi, porque el modelo de investigación de audiencia de los medios radiotelevisivos puede considerarse prácticamente muerto, habiendo triunfado paradójicamente la forma panel de seguimiento de las audiencias, ya no sólo como seguimiento de sus movimientos, sino que son 
seguidas y capturadas por máquinas inteligentes (machine learning) que les van adaptando los mensajes.

Conceptos que venían del audímetro, de la cultura del audímetro, hoy dejan de tener sentido, como el de share o cuota de pantalla; puesto que la visión de un programa o contenido se extiende en el tiempo, siendo varios los momentos potenciales. ¿Qué significa hoy pantallas en un momento determinado? Hasta hace poco, los programas se producían en directo o en diferido; mientras que las audiencias siempre eran producidas en directo. Hoy también se producen audiencias en diferido, en otros momentos y con la participación de otras audiencias, que llaman la atención sobre un mensaje, que intentan redireccionar a los miembros de sus social media.

\section{Más allá de los debates sobre la medida, la recomposición del sistema de comunicación mediada}

La medición de audiencia es propia y funcional del modelo de relación entre sistema social de mercado y sistema de comunicación programada de "inserción de anuncio" en un modelo de difusión masiva ¿va a seguir este modelo? ¿Cómo funcionaba el mercado, el sistema de mercado, antes del surgimiento de la publicidad masivocorporativa?

- Eran los distribuidores - concretamente los dependientes en las tiendas- los que directamente instruían o se imitaba a las clases altas (a la clase ociosa, de Veblen, 2014), cumpliendo así una de sus funciones, servir de imitación (Bourdieu, 1988)

- Con la publicidad masivo-corporativa incluso las clases altas se instruían sobre lo que consumir a partir de la publicidad y los distribuidores (pequeños) tendieron a desaparecer. El consumidor ya estaba suficientemente educado para estar en contacto con los productos y servicios tras el mensaje, sin necesidad de mediadores.

- En la actualidad: a) los instructores son influencers, bloggers, instagramers o facebookers, y, en general, están en las redes o internet, a partir de la capa de expertos o comunidad de usuarios; b) los propios anunciantes son medios, a través de sus web y de remitir publicidad directamente a los clientes utilizando las redes sociales; c) la publicidad se establece en la creación de acontecimientos (deportivos, culturales, etc.), como base de lo informativo, de manera que tiende a seguirse solo aquello que es susceptible de convertirse en soporte de publicidad.

Siguiendo a Luhmann (2000) las operaciones del sistema de comunicación mediada no necesitan de la medida, lo que necesitan es asegurar que hay recepción, que los mensajes o las acciones de los medios tienen recepción. No cabe duda de que, con la medida, se tiene un espejo que indica lo que tiene más y menos recepción; pero el acento en la misma, en tal medida, es más del sistema de mercado, de los anunciantes. Puede decirse que el sistema de medios de comunicación se conforma con la sombra de la recepción, suficiente para diseñar su planificación, pues lo que hace el sistema en el modelo tradicional de radiodifusión es programar. Sin embargo, los anunciantes exigen unidades de actores en pos de sus consumos y servicios. 
Más allá de la programación, el sistema de comunicación mediada selecciona la información y la categoríza. Produce y procesa la información y de esta manera, convierte la información en no información; por lo que el sistema se ve obligado a obtener (nueva) información, más información. Los medios, permanentemente desactualizan la información ${ }^{21}$, lo que genera, a su vez, una permanente demanda de información. Para satisfacer tal demanda, antes había que esperar a la hora del informativo. Así, se acumula audiencia en un período determinado del día ${ }^{22} \mathrm{y}$, sobre todo, con tal fin se diseña la programación. En la actualidad, esa demanda permanente se satisface principalmente también de forma permanente. Las elecciones de la fuente de información por parte de los receptores ya no derivan de la hora de emisión del programa, frente al que se acumula la audiencia, sino de la confianza que se tiene en la fuente, en su reputación. La selección de lo informativo ha de ser confiable, pues ya no está programado.

La programación tiene sentido en una forma de emisión/recepción centralizada, bajo el modelo de un solo foco de emisión que irradia sus mensajes a toda la audiencia potencial. Pero esto explota por los aires cuando son cientos de miles los emisores en cada instante con internet. Aun cuando hay sistemas -Google, Facebook- que aún sostienen la centralización, concentrando convergentemente a los emisores y, así, acumulando toda la audiencia y sus comportamientos de una manera prácticamente monopolista, se abren modelos alternativos descentralizados que establecen la directa relación entre múltiples emisores y múltiples receptores. El modelo dominante actual, basado en esa lógica de concentración subordinada de los emisores, es el que está a punto de acabar económicamente con el modelo tradicional de los medios de comunicación, ya que el punto de convergencia -por ejemplo, Google-es el que se lleva el grueso de los ingresos por publicidad ${ }^{23}$; de manera que parece que los propios emisores trabajan para tal punto de concentración de los ingresos publicitarios.

La medición de audiencias, como acoplamiento estructural -utilizando el concepto de Luhmann (2007)- entre sistema de mercado y sistema de comunicación programada, parece tener menor relevancia. En especial porque el sistema social de mercado cada vez necesita menos de la mediación del sistema de comunicación programada para concentrar la atención de los consumidores. Como se ha señalado, se multiplican las posibilidades de emisión, de convertirse en medios de comunicación -incluso en el modelo de concentración por encima en un punto, son múltiples los emisores- con el hecho de que el mismo anunciante se convierte en un medio. Basta con acudir a algunos sitios web corporativos y cómo se emplea la misma lógica que los medios tradicionales utilizan para atraer la atención de la audiencia: generan expectativas, de que "algo nuevo puede pasar" (Luhmann, 2000:83). Tal vez, por ello, se especializan en lo nuevo, en ser vehículo para anunciar el futuro anunciándose ellos, sobre cómo nos va a cambiar el mundo, como ocurre en los sitios corpora-

${ }_{21}$ Por eso la publicidad molesta, porque es repetitivo, no da información. (el modelo que parece tener Luhmann es el del dinero, el de la economía, si gastas más dinero, tendrás que trabajar para obtener más dinero). Dices lo que es nuevo, información, y lo que es viejo. Cuando algo es designado como nuevo, se está designando el resto como viejo. La principal función de los medios de comunicación, como sistema, es diferenciar entre lo nuevo y lo viejo; marcar lo que es nuevo y lo que es viejo.

22 Como subrayan las divisiones del día en función de las expectativas de acumulación de audiencia: prime time, second time...

23 Visto desde su perspectiva, Google -o Facebook- utiliza a las redes sociales, especialmente algunos de sus perfiles más activos, como trampolín para acceder a una audiencia mucho más amplia. 
tivos de bancos u operadoras telefónicas. Utilizan la misma táctica que el periodismo para crear adicción. Para ellos, trabaja un periodismo de serial, una manera de informar para la suscripción y lograr que los receptores se mantengan en la página. Se va a promover el carácter secuencial de los productos mediáticos para mantener audiencia. A veces, incluso, creando los acontecimientos que son reportados para, así, sostener publicidad. Pero son los propios anunciantes los que sostienen el acontecimiento, desde la Liga Santander, al open Mapfre de tenis de Madrid, por solo referirnos al campo del deporte. Es la publicidad la que genera o sostiene el acontecimiento, según los casos, y no, como venía ocurriendo, el acontecimiento como soporte para la publicidad.

Por último, señalar que la relación entre emisores y receptores no tiene que pasar forzosamente por la publicidad. Al menos, por la publicidad centralizada. Parece difícil distanciarse de un modelo que ha dominado tan intensamente el sistema de medios de comunicación; pero se abren alternativas descentralizadas, como es la posible monetización directa de los contenidos, por parte de los emisores, a partir de la tecnología blockchain. Los creadores de contenido reciben micropagos por sus trabajos directamente de los consumidores (Fernández, 2017) y, a la vez, esta tecnología posibilita un seguimiento de los usos de sus obras, dificultando el denominado pirateo y la pérdida de control sobre las copias y reproducciones de sus trabajos. ¿Qué papel, más allá del centrado en la investigación básica y, en líneas generales, académica, tendría entonces la investigación de audiencias? No hay "audiencia que vender", pues no hay publicidad.

\section{A modo de conclusión: retomar el rumbo}

La presentación de los retos con los que se enfrenta en la actualidad la investigación de audiencias aplicada ha tenido por objetivo abrir un marco para la reflexión sobre la necesidad de que este tipo de investigación tome nuevos rumbos. Hasta ahora, ha estado subordinada a un contexto dominado por la relación entre sistema social de mercado y sistema social de los medios de comunicación, centrado en la publicidad difundida masivamente. Con esto no se quiere decir que la publicidad no siga siendo importante, pues lo seguirá siendo el consumo. Pero su funcionamiento parece destinado a ser distinto a como lo ha sido en los últimos decenios. La racionalización de la publicidad continuará; pero llevando otros caminos, ya sea ajustándose algorítmicamente a los perfiles tomados de los consumidores a partir de la acumulación de los registros que deja su continuo paso por internet, como asegura el centralizado modelo big data, ya que solo se puede acumular desde la concentración de datos bajo la centralización; ya sea bajo modelos más descentralizados, en el que se busca la participación activa e implicación de la audiencia con la identificación de los contenidos, incluyendo los contenidos publicitarios. Según esta polaridad de modelos, en ninguno de ellos parece tener sentido la tradicional investigación de audiencias, pues, desde el primero, para qué buscar los perfiles sociodemográficos de los consumidores de medios, de la audiencia, si se tienen sus perfiles de intereses, de lo que llama su atención, de lo que buscan y, tal vez lo más importante, es posible el directo acceso a ellos cada vez que encienden determinadas pantallas. Desde el segundo, la investigación de audiencias adquiere otro sentido, adquiere la función de generar información para activar y transformar comunidades, tomando más el papel de un tipo de investigación 
presente en otros campos, pero prácticamente inédita en el campo de los medios de comunicación, como es el de la investigación-acción participativa.

Una audiencia para la participación y una investigación para generar comunidad, aun cuando sea una comunidad de seguimiento o, haciéndonos eco de los trabajos de Jenkins (2010), comunidad de fans, de seguidores, cambia radicalmente el concepto de audiencia. Sobre todo, rompe con su concepción como mercancía, como valor de cambio. La investigación de la audiencia habrá de centrarse en el valor de uso de la audiencia, en su participación, en qué hacer con ella y para ella. Tal vez, así, retomando de forma diferente a la audiencia, la investigación de audiencia deje de estar tan perdida como lo está desde que se expandió la transición digital.

\section{Referencias bibliográficas}

Adorno, T.W., y Horkheimer, M. (1971): Dialéctica del Iluminismo, Buenos Aires, Editorial Sur.

Barberá, P. (2015): "Birds of the Same Feather Tweet Together. Bayesian Ideal Point Estimation Using Twitter Data", Political Analysis, 23 (1), pp. 76-91.

Bermejo, F. (2007): The Internet Audience: Constitution and Measurement, Nueva York, Peter Lang.

Bell, D. (1973): El advenimiento de la sociedad post-industrial, Madrid, Alianza.

Boltanski, L., y Esquerre, A. (2017): Enrichissement. Une Critique de la Marchandise, París, Gallimard.

Bourdieu, P. (1988): La distinción, Madrid, Taurus.

Burke, P. (2017): Historia social del conocimiento. De Gutenberg a Diderot, Barcelona, Austral.

Callejo, J (1995). "La investigación cualitativa de los medios de comunicación”. Investigación y marketing, ISSN 1131-6144,47, págs.57-62.

Callejo, J. (2000). "Notas sobre la concepción de modo por los manuales de Técnicas de Investigación Social”. Empiria: Revista de metodología de ciencias sociales, ISSN11395737,3, 2000, págs.195-208

Callejo, J. ( 2018). Investigar las audiencias: un análisis cualitativo. Barcelona: Paidós.

Carr, N. (2011): The Shallows: What the Internet Is Doing to Our Brains, Nueva York, W. W. Norton \& Company.

Deacon, D., y Keigthley, E. (2011): “Quantitative Audience Research: Embracing the Poor Relation", en V. Nightingale, Handbook of Media Audiences, Oxford, Wiley-Blackwell, pp. 302-319.

Domingos, P. (2015): The Master Algorithm, Nueva York: Basic Books.

Eco, U. (1982): “¿El público perjudica a la televisión?”, en M. de Moragas (ed), Sociología de la comunicación de masas, Barcelona, Gustavo Gili.

Fernández, C. (2017): "Medios de comunicación y la blockchain”, en A. Preukschat, Blockchain: la revolución industrial de internet, Barcelona, Gestión 2000, pp. 83-88.

Finn, E. (2017): What algorithms want, Cambridge (Mass.), MIT Press.

García Matilla, E. (1990): Subliminal: escrito en el cerebro, Madrid, Bitácora.

Gitlin, T. (2000): Inside Prime Time, Berkeley, University of California Press, e.o. 1983.

Gitlin, T. (2007): Media Unlimited: How the Torrent of Images and Sounds Overwhelms Our Lives, Nueva York, St. Martin's Press.

Hadnagy, CH. (2010): Social Engineering: The Art of Human Hacking, Nueva York, John Wiley \& Sons. 
Huertas, A. (2002): La audiencia investigada, Barcelona, Gedisa.

Huertas, A. (2015): Yo soy audiencia: ciudadanía, público y mercado, Barcelona, UOC.

Ibáñez, J. (1979): Más allá de la sociología, Madrid, Siglo XXI.

Jenkins, H. (2010): Piratas de textos. Fans, cultura participativa y televisión, Barcelona, Paidós.

Jenkins, H., FORD, S., y GREEN, J. (2015): Cultura Transmedia. La creación de contenido $y$ valor en una cultura en red, Barcelona, Gedisa.

Katz, E. (1987): “Communication reaserach since Lazarsfeld”, Public Opinion Quarterly 51 (4), pp.25-45.

Kelleher, J.d., Mac Namee, B., y D'arcy, A. (2015): Fundamentales of machine learning for predictive data analytics: algorithms, worked examples, and case studies, Cambridge (Mass.), MIT Press.

Klapper, J.T. (1974). Efectos de la comunicación de masas, Madrid, Aguilar (e.o. 1960).

Lazarsfeld, P.F. (1940): Radio and the Printed Page, Nueva York, Duell, Sloan y Pearce.

Lazarsfeld, P.F. (1941): "Remarks on administrative and critical communications research", Studies in Philosophy and Science 9, pp. 3.16.

Lazarsfeld, P.F. (1950): “The obligations of the 1950 pollster to the 1984 historian”, Public Opinion Quarterly 14, pp. 618-638.

Lazarsfeld, P.F. (1972): Qualitative Analysis, Boston, Allyn and Bacon.

Lazarsfeld, P.F., y KENDALL, P. (1948): Radio Listening in America, Englewood Cliffs (NJ), Prentice-Hall.

Lazarsfeld, P.F., y Merton, R.K. (1948): "Mass communication, popular taste and organized social action", en L. Bryson (ed.), Communication of ideas, Nueva York, Harper and Row.

Lazarsfeld, P.f., y Stanton, F.N. (eds.) (1942): Radio Research 1941, Nueva York, Duell, Sloan and Pearce.

Lazarsfeld, P.f., y Stanton, F.N. (eds.) (1944): Radio Research 1942-43, Nueva York, Duell, Sloan and Pearce.

Lazarsfeld, P.f., y Stanton, F.N. (1949): Communcation Research 1948-49, Nueva York, Duell, Sloan and Pearce.

Lazarsfeld, P.f., Berelson, B., y Gaudet, H. (1944): The People's Choice, Nueva York, Duell, Sloan and Pearce.

Luhmann, N. (1990): Essays on Self-Reference, Nueva York, Columbia University Press.

Luhmann, N. (2000): La realidad de los medios de masas, Barcelona, Anthropos Y Universidad Iberoamericana.

Luhmann, N. (2007): La Sociedad de la Sociedad, México, Herder-Universidad Iberoamericana.

Marres, N. (2017): Digital Sociology. The Reinvention of Social Research, Oxford, Polity Press.

Mitnick, K. (2011): Ghost in the Wires: My Adventures as the World's Most Wanted Hacker, Boston, Little, Brown and Company.

Napoli, PH. (2011): "Ratigns and Media Measurement" en V. Nightingale, Handbook of Media Audiences, Oxford, Wiley-Blackwell, pp. 286-301..

Nightingale, V. (1996): Studying Audiences. The shock of the real, Routledge, Londres.

O'neil, C. (2016): Weapons of Mass Destruction: How Big Data Increases Inequality and Threatens Democracy, Nueva York: Random House.

Ogilvy, D. (1984): Confesiones de un publicitario, Barcelona, Orbis.

Packard, V.O. (1957): The Hidden Persuaders, Nueva York, Random House. 
Schiller, H.I. (1983): “La comunicación sigue al capital”, en G. Richeri (ed), La televisión: entre servicio público negocio, Barcelona, Gustavo Gili, pp. 51-70.

Simon, H.A. (1996): The Sciences of the Artificial, Cambrige y Londres, MIT Press (e.o. 1981).

Smythe, D.W. (1981): “On the Audience Commodity and Its Works" en Dependency Road: Communicationss, Capitalism, Consciousness, and Canada, Norwood, Ablex, pp. 22-51.

Smythe, D.W. (1983): "Las comunicaciones: 'agujero negro' del marxismo occidental”, en en G. Richeri (ed), La televisión: entre servicio público negocio, Barcelona, Gustavo Gili, pp. 71-103.

Van Zoonen, W., y Toni, G.L.A. (2016): "Social media research: The application of supervised machine learning in organizational communication research", Computers in Human Behavior, 63, pp.132-141.

Veblen, T. (2014): Teoría de la clase ociosa, Madrid, Alianza, e.o. 1899.

Vecchiato, G., Cherubino, P., Trettel, A., Babiloni, F. (2013): Neuroelectrical Brain Imaging Tools for the Study of the Efficacy of TV Advertising Stimuli and their Application to Neuromarketing, Berlín-Heidelberg, Springer-Verlag.

Williams, R. (1974): Television: Technology and Cultural Form, Nueva York, Schocken.

Wright Mills, Ch. (1999): La imaginación sociológica, Madrid, Fondo de Cultura Económica. 